\title{
Corela
}

Cognition, représentation, langage

14-2| 2016

Vol. $14, \mathrm{n}^{\circ} 2$

\section{Retour sur les annotations des entités nommées dans les campagnes d'évaluation françaises et comparaison avec la TEI}

Solenn Le Pevedic et Denis Maurel

\section{(2) OpenEdition}

\section{Journals}

Édition électronique

URL : http://journals.openedition.org/corela/4644

DOI : $10.4000 /$ corela.4644

ISSN : 1638-573X

Éditeur

Cercle linguistique du Centre et de l'Ouest - CerLICO

Référence électronique

Solenn Le Pevedic et Denis Maurel, « Retour sur les annotations des entités nommées dans les campagnes d'évaluation françaises et comparaison avec la TEI », Corela [En ligne], 14-2 | 2016, mis en ligne le 08 décembre 2016, consulté le 30 avril 2019. URL : http://journals.openedition.org/ corela/4644; DOI : 10.4000/corela.4644

Ce document a été généré automatiquement le 30 avril 2019.

\section{(c) (i) (3)}

Corela - cognition, représentation, langage est mis à disposition selon les termes de la licence Creative Commons Attribution - Pas d'Utilisation Commerciale - Partage dans les Mêmes Conditions 4.0 International. 


\title{
Retour sur les annotations des entités nommées dans les campagnes d'évaluation françaises et comparaison avec la TEI
}

\author{
Solenn Le Pevedic et Denis Maurel
}

\section{Introduction}

1 La notion d'entité nommée est apparue lors des conférences Muc (Message Understanding Conference) aux États-Unis. Le but de ces conférences était d'évaluer les systèmes d'extraction d'informations. La définition est donnée par (Chinchor, 1997) pour la conférence Muc-7 : On the level of entity extraction, Named Entities (NE) were defined as proper names and quantities of interest. Person, organization, and location names were marked as well as dates, times, percentages, and monetary amounts. Cette notion a été étudiée et affinée du point de vue linguistique par (Ehrmann, 2008) : Étant donné un modèle applicatif et un corpus, on appelle entité nommée toute expression linguistique qui réfère à une entité unique du modèle de manière autonome dans le corpus. Le lien qu'elle fait entre modèle applicatif et corpus introduit la question abordée dans cet article, à savoir la portabilité des annotations en entités nommées.

Pour comparer les résultats obtenus par différentes équipes de Tal, l'idée est de définir des annotations à insérer dans un corpus et d'évaluer les résultats de chaque équipe, à la fois en terme de précision (a-t-on annoté correctement une entité ?) et de rappel (en a-ton oublié certaines ?).

Une campagne d'évaluation propose donc des annotations à insérer dans un corpus sur lequel tous les systèmes participants seront évalués en terme de rappel et précision (et/ ou F-mesure). Un guide d'annotation précise le résultat attendu. Il est accompagné d'un corpus d'entraînement et de test, notamment pour les systèmes à base d'apprentissage. 
Cette annotation manuelle est elle-même contrôlée par des mesures d'accord interannotateurs (Artstein et Poesio, 2008) (Fort et al., 2012). Depuis la conférence Muc-7 qui portait sur l'anglais, d'autres campagnes ont eu lieu sur d'autres langues, comme, entre autres, Met (Merchant et al., 1996) pour le japonais, le chinois et l'espagnol, Irex (Sekine et Eriguchi, 2000) pour le japonais, Harem (Santos et al., 2006) pour le portugais, etc. Nous tenons aussi à rappeler les campagnes Language-Independent Named Entity Recognition des conférences CoNLL-2002 $2^{1}$ et CoNLL-20032 , entièrement basées sur l'apprentissage.

Pour le français, deux ${ }^{3}$ campagnes ont eu lieu sur des corpus oraux transcrits, soit manuellement, soit automatiquement. Ces campagnes, organisées sous l'égide de l'association francophone de communication parlée (AFCP), ont eu lieu en 2008 pour Ester-2 et en 2012 pour Etape. La campagne Ester-2 utilisait un guide d'annotation (Ester-2, 2007), testé lors de la campagne Ester-1, puis revu et disponible en ligne. La campagne Etape s'est appuyée sur le guide d'annotation défini dans le cadre du projet Quaero (Rosset et al., 2011).

Le principal inconvénient des annotations préconisées dans ces deux campagnes est leur manque de portabilité. Il est nécessaire de refaire les corpus d'apprentissage et d'adapter les systèmes à base de règles. La présentation des résultats et des exemples n'est pas simple en dehors du cercle restreint des participants ou, du moins, en comparaison avec d'autres systèmes sur d'autres langues.

6 Parallèlement aux guides d'annotation sur les entités nommées, un consortium, la TEI ( Text Encoding Initiative $e^{4}$ issu de différentes disciplines en sciences humaines a travaillé, dès la fin des années 1980, sur un projet de définition d'un balisage standard des documents dans un format électronique. Les documents concernés pouvant tout aussi bien être du texte, de l'image, de la vidéo, du son, etc. avaient pour but de faciliter les échanges internationaux de documents. Des années de recherche ont été nécessaires avant de pouvoir publier le premier guide en 1994. Depuis cette date, celui-ci n'a pas cessé d'évoluer. La version actuelle en est la cinquième version couramment appelée TEI P5 (Text Encoding Initiative Consortium, 2015). À l'origine, la TEI suivait la norme SGML, mais aujourd'hui elle utilise le langage XML. Le balisage ne concerne pas seulement le texte lui-même, car la TEI préconise aussi le balisage des informations liminaires que l'on peut obtenir sur le document à encoder (titre, auteurs, éditeurs, résumé, pagination, paragraphes, etc.). La TEI permet aussi de baliser la structure du texte lui-même (paragraphes, phrases, mots...).

7 L'annotation temporelle existe dans la TEI, mais sur ce point précis, d'autres travaux ont proposé des guides de balisage, citons la thèse d'Andrea Setzer (Setzer, 2001), le projet Tides (Ferro et. al., 2001) et, pour finir, TimeML (Pustejovsky, 2003). Ce dernier est devenu une norme ISO, définie en mars 2009. TimeML concerne non seulement les expressions temporelles, souvent considérées comme des entités nommées, mais aussi les évènements portés par les prédicats, ce qui sort du contexte de cet article ${ }^{5}$.

8 L'article s'articule autour de trois parties: en section 1, nous faisons un retour sur les conventions des campagnes Ester et Etape. Dans la section 2, nous présentons les balises TEI concernant les entités nommées. Enfin, dans la section 3 nous préconisons des solutions pour remplacer les balises du projet Quaero par des balises TEI. 


\section{Retour sur les campagnes Ester et Etape}

\subsection{Les campagnes Ester-1 et Ester-2}

9 radiophoniques (plus communément appelée Ester) avaient pour but d'évaluer la performance des systèmes sur différents types de tâches, essentiellement la transcription et l'indexation d'émissions de radio. La campagne se divise en deux phases successives. La première phase courait de 2003 à 2005, la seconde de 2006 à 2008. Plusieurs tâches sont effectuées lors de ces campagnes, telles que la segmentation et la transcription orthographique, mais, également, ce qui nous intéresse ici, la reconnaissance des entités nommées.

La première phase n'exploite pas totalement cette tâche contrairement à la seconde dont il s'agit d'un des thèmes principaux. Elle est principalement soutenue par l'Association francophone de la communication parlée (AFCP), mais aussi par le Centre d'expertise parisien de la Délégation générale pour l'armement (DGA/CEP) et par l'European Language Ressources Association (ELRA) qui permet, quant à elle, la pérennité du projet par la diffusion de corpus annotés.

11 Un corpus conséquent a donc été établi. Celui-ci contient des articles de presse tirés du journal «Le Monde », des textes tirés des débats du Conseil européen, et, surtout, d'une centaine d'heures d'émissions de radio (principalement France Inter) transcrites pour et par cette tâche. Le corpus entre la première et la seconde phase a été légèrement remanié. Le nombre d'heures de ressources orales transcrites passent de $78 \mathrm{~h} 55$ à $103 \mathrm{~h} 08$. Pour ce corpus, on recense environ 150000 entités nommées pour ce corpus.

\subsection{Retours sur le guide de la campagne Ester-2}

Le guide d'Ester-2 qui est une version revue et augmentée de celui de la campagne Ester-1, est disponible en ligne sur le site de l'AFCP. Pour ce guide, les entités nommées sont au cœur de la problématique de l'extraction de l'information d'un document. Ce sont des types particuliers d'unités lexicales (groupes de mots) qui font référence à une entité $\mathrm{du}$ monde concret dans certains domaines spécifiques, notamment: humains, sociaux, politiques, économiques ou géographiques et qui ont un nom (typiquement un nom propre ou un acronyme). S'ajoutent à la tâche de reconnaissance des entités nommées celle des informations « temps » et « montant».

En dehors des personnes, lieux et organisations déjà présents dans la campagne Muc-7, on retrouve deux nouveaux types: les fonctions (politiques, militaires, administratives, religieuses et aristocratiques) et les productions humaines (moyens de transport, récompenses, œuvres artistiques et productions documentaires). Ces deux types supplémentaires semblent contredire la précision donnée en introduction du guide et rappelée ci-dessus, qui stipule que les entités nommées correspondent à des entités ayant un nom, même si ce sont bien des entités du monde concret. Pour les fonctions, comme dans l'exemple ${ }^{6}$, il s'agit bien de personnes qui sont ainsi désignées.

14 1. Le <ent type ="fonc.admi">vice-directeur $</$ ent $>$ est mis en examen suite à l'affaire... 

produit : est-ce un nom propre ou un nom commun? (Fèvre-Pernet, Roché, 2005) ou une classe d'objets? (Gross, 2012). L'exemple semble désigner la classe, alors que l'exemple semble plutôt correspondre à un objet particulier. que les deux fonctions (député/grandrabbin) sont associées à (UMP/ashkénaze) qui précisent le type de député et le type de grand rabbin.

4. $\quad$ ent type $="$ pers.hum" $><$ ent type $="$ pers.hum" $>$ Pierre $\quad$ Lellouche $</$ ent $>$ le $<$ ent type $=$ "fonc.pol">député UMP de <ent type $=$ "loc.admi" $>$ Paris $</$ ent $></$ ent $></$ ent $>$

5. le <ent type $=$ "pers.hum"><ent type $=$ "fonc.rel" $>$ grand rabbin</ent $>$ ashkénaze $<$ ent type $=$ "pers.hum" $>$ Avraham Shapira $</$ ent $></$ ent $>$

Il nous semble qu'il faudrait plutôt écrire <ent type ="fonc.rel">grand rabbin ashkénaze</ ent>.

Ajoutons à cela une remarque sur des difficultés quasiment insurmontables (sans connaissances pragmatiques ou encyclopédiques). Si il est judicieux de séparer, pour un même nom, l'aspect lieu de l'aspect institution, comme les exemples et, car le contexte permet En dehors des personnes, lieux et organisations déjà présents dans la campagne Muc-7, on retrouve deux nouveaux types: les fonctions (politiques, militaires, administratives, religieuses et aristocratiques) et les productions humaines (moyens de transport, récompenses, oeuvres artistiques et productions documentaires).la recherche d'entité nommée par ce qu'on appelle aujourd'hui l'entity linking (McNamee et al., 2010). 6. Je suis stationné à côté de la <ent type ="loc.fac">mairie de Paris</ent>.

7. La course à la <ent type ="org.pol">mairie de Paris</ent $>$ a commencé.

8. Je me suis fait opérer à l'<ent type ="org.non-profit">hôpital Necker</ent>.

Enfin terminons par la décision de ne pas étiqueter les lieux personnels, c'est-à-dire tout lieu ou habitation désignée comme appartenant à un particulier. Comment savoir si, dans l'exemple , la dite propriété est réellement privée ou est un lieu public, même si le propriétaire est privé ? Citons l'exemple du château de Villandry qui est une propriété privée ouverte à la visite, comme un musée, et dans laquelle le propriétaire habite !

9. La propriété Saint Vincent a été rachetée par le Comte de Bourgogne

\subsection{La campagne Etape}

La campagne Etape émane du projet européen Quaero 8 qui s'est déroulé sur une période allant de 2008 à fin 2013. Elle se proposait d'évaluer les résultats de ce projet en termes de recherche d'entités nommées. Cependant, cette campagne d'évaluation a été ouverte largement et des équipes ne participant pas au projet Quaero ont pu y contribuer.

C'est dans le cadre de ce projet qu'un guide d'annotation a été réalisé (Rosset et al., 2011). La complexité des annotations a largement augmenté (Table 1). 
Table : Nombre d'étiquettes et de pages des guides d'annotation

\begin{tabular}{|l|l|l|}
\hline Campagne & Nombre d'étiquettes & Nombre de pages \\
\hline Muc-7 & 7 & 23 \\
\hline Ester-2 & 37 & 25 \\
\hline Etape & 54 & 86 \\
\hline
\end{tabular}

\subsection{Retours sur le guide de la campagne Etape}

Comme cela était évoqué dans les précédents guides, la notion d'entités nommées recouvre (entre autres) celle de noms propres. Le guide Quaero s'affirme en cohérence avec la définition des entités nommées de la campagne Ester-2. Il adopte:

- la définition des entités nommées de la thèse de Maud Ehrmann (2008);

- la définition des entités temporelles du projet Time ML (Pustejovsky, 2003) ;

- la taxinomie de la thèse de Mickaël Tran (Tran, 2006 ; Tran et Maurel, 2006);

- la hiérarchie des entités nommées étendues proposée par Satoshi Sekine (Sekine,2004, Nadeau and Sekine, 2007).

Comme le projet Quaero se place dans le contexte d'extraction d'informations, il demande l'inclusion d'expressions ne contenant pas de nom propre et étend l'annotation à des expressions construites autour de noms communs, comme sur l'exemple .

10. $<$ func.ind $>$ extractor $>$ un $</$ extractor $>$ des $<$ kind $>$ pompiers $</$ kind $></$ func.ind $>$

Le guide est très complet et comporte de nombreux exemples. L'annotation n'est plus uniquement centrée sur les entités, mais aussi sur les composants de ces entités, comme on le voit sur ce même exemple .

Les composants principaux sont <name> pour le nom de l'entité et <kind $>$ ou <qualifier> pour les noms communs et les qualifieurs constituant l'entité étendue. Nous ne ferons qu'une remarque sur ces annotations internes, concernant la distinction entre les qualifieurs suivant qu'ils sont intégrés ou non à des expressions temporelles, comme sur les exemples et .

11. le <prod.rule $>$ <ualifier $>$ dernier $<$ /qualifier $>\quad<$ kind $>$ plan de paix $<$ / kind $><$ qualifier $>$ international $<$ qualifier $></$ prod.rule $>$

12. $<$ time.hour.abs $><$ time-modifier $>$ aux alentours de $</$ time-modifier $><$ val $>$ quinze $</$ val $>$ $<$ unit $>$ heures $</$ unit $><$ val $>$ trente $</$ val $></$ time.hour.abs $>$

Or dernier sera annoté time-modifier dans le dernier jeudi du mois et aux alentours de sera annoté qualifier dans aux alentours de $15 \mathrm{~kg}$. Bien sûr, cela vient probablement du fait que certains qualifieurs ne peuvent concerner les expressions temporelles, comme socialiste dans l'exemple, et inversement, certains modifieurs ne concernent que celles-ci, comme 
matin dans l'exemple. Il nous semble cependant que ces deux étiquettes auraient dû être confondues. absolues et relatives. Dans les campagnes Ester, une date est considérée absolue lorsque la date du document annoté n'est pas nécessaire pour la déterminer précisément et, a contrario, une date est considérée comme relative lorsque la date du document annoté est indispensable pour la déterminer précisément. Dans le guide Quaero, deux sortes de dates absolues sont considérées, les dates absolues précises (celles de la campagne Ester) et les dates absolues imprécises dont le point de référence dans le calendrier n'est pas connu avec certitude mais dont les composants sont absolus. Par contre, dès qu'un marqueur explicite de relativité (par exemple, prochain) est spécifié (rendez-vous mardi prochain), on a une date relative. déclarer un rendez-vous mardi ou mardi prochain? Et le changement de déterminant dans un rendez-vous le 25 janvier (date absolue) et un rendez-vous ce 25 janvier (date relative) crée-t-il réellement une différence sémantique?

\section{Les entités nommées dans la TEI}

\subsection{Les éléments les plus généraux}

L'élément le plus général qu'il est possible d'utiliser est la balise referencing string qui indique le renvoi à un référent. De ce fait, cet élément peut baliser un nom propre, mais aussi toute référence anaphorique y compris pronominale.

17. My dear $<\mathrm{rs}$ type="person">Mr. Bennet $</ \mathrm{rs}>$, said $<\mathrm{rs}$ type="person">his lady $</ \mathrm{rs}>$ to him one day

Plus spécifiquement, mais de manière encore très générale, l'élément name permet le balisage des noms propres .

18. $<$ name type="person">Mr. Bennet $</$ name>

19. $<$ name type $=$ "place" $>$ Netherfield Park $</$ name $>$

Ces balises peuvent contenir des attributs permettant de lier des entités (id, pour un identifiant unique, key, pour une clé et ref pour le référent), mais ceci n'était pas considéré par le guide Quaero.

Le terme entité apparaît dans le guide TEI dans la sous-classe d'attributs att.naming. On y trouve par exemple l'attribut role qui donne des informations spécifiques sur l'entité encodée: @role may be used to specify further information about the entity referenced by this name, for exemple the occupation of a person, or the status of a place.

20. $<$ name role="politician" type="person">David Paul Brown $</$ name $>$ 


\subsection{Personnes, lieux et organisations}

Le guide TEI introduit aussi des éléments plus spécifiques concernant les personnes, lieux et organisations.

L'élément persName permet le balisage des noms de personne. Il est accompagné de plusieurs autres éléments permettant de spécifier le prénom (forename) ou le nom ( surname), mais aussi les titres (roleName), les surnoms (addName), les générations (genName ) ou même les particules (nameLink) ou la notion d'abréviation (full)... .

21. $<$ persName $><$ roleName type="nobility" $>$ Princess $</$ roleName $>$ forename $>$ Grace $<$ / forename $><$ /persName $>$

22. $<$ persName $><$ roleName type $=$ "office" $>$ Governor $</$ roleName $><$ forename $>$ Edmund $</$ forename $>$ forename full="init" $>$ G. $</$ forename $>$ addName type="nick" $>$ Jerry $<1$ addName $>$ addName type="epithet" $>$ Moonbeam $</$ addName $>\quad<$ surname $>$ Brown $<$ / surname $><$ genName full="abb">Jr $</$ genName> $></$ persName $>$

Les lieux sont divisés en deux, les lieux administratifs (placeName) et les lieux géographiques (geogName) . Les lieux géographiques sont spécifiés par l'élément geogFeat.

23. <placeName>New York</placeName>

<geogName type="river"><name>Mississippi</name>

24. <geogFeat $>$ River $<$ /geogFeat $><$ /geogName $>$

La TEI introduit ici la notion de lieu relatif (relative place), c'est-à-dire un lieu qui est défini par sa situation relativement à un autre lieu, par deux éléments, offset et measure. Le premier va marquer une direction (nord, sud...) et le second une distance .

25. < placeName $><$ measure $>20 \mathrm{~km}<$ /measure $><$ offset $>$ north of $</$ offset $><$ settlement type="city" $>$ Paris $</$ settlement $></$ placeName $>$

Enfin, il existe aussi dans le guide, une description très complète des adresses postales, avec l'élément address qui peut contenir divers autres éléments, comme street, postCode, settlement, country, etc. . Cependant il est aussi possible de ne pas spécifier ces éléments internes et de les remplacer par un seul élément, addrLine.

26. $<$ address $><$ street $>$ via Marsala 24</street $><$ postCode $>40126</$ postCode $><$ settlement type="city" $>$ Bologna $<$ /settlement $><$ country $>$ Italy $</$ country $></$ address $>$

27. <address $><$ addrLine $>$ Computing Center, MC 135</addrLine $><$ addrLine>P.O. Box 6998</addrLine> <addrLine>Chicago, IL 60680</addrLine> < addrLine>USA</addrLine></ address $>$

La notion d'adresse électronique est aussi présente, avec l'élément email .

28. <email>info@tei-c.org</email>

Quant aux organisations, c'est l'élément orgName qui est préconisé par le guide TEI : We use the term " organization " for any named collection of people regarded as single unit.

29. $<$ orgName type="voluntary">Pennsyla. Abolition Society</orgName>

\subsection{Dates, temps, mesures}

Les valeurs numériques de toute sorte peuvent être encodées : pourcentages, fractions, nombres cardinaux, romains, chinois... éventuellement avec un attribut value . 

chercher si il existe ou non une correspondance dans la TEI. Les exemples reprennent dans l'ordre un grand nombre ${ }^{10}$ de ceux du guide Quaero.

\subsection{Personnes}

79 À quelques différences près sur le balisage interne, les exemples de Quaero se transposent bien en TEI. Les éléments function, qualifier et extractor peuvent être remplacée respectivement par les éléments roleName, addName, complété par l'attribut epithet, et num, complété par l'attribut ordinal .

80 35. $<$ pers.ind $><$ title $>$ Sa Majesté le $<$ func.ind $>$ roi $</$ func.ind $></$ title $><$ name.first $>$ Mohamed $</$ name.first $><$ qualifier $>\mathrm{VI}</$ qualifier $></$ pers.ind $>$

$$
<\text { persName }>\text { addName type }=\text { "honorific" }>\text { Sa } \quad \text { Majesté }</ \text { addName }>\text { le }<\text { roleName }
$$


type $=$ "nobility" $>\quad$ roi $</$ roleName $><$ forename $>$ Mohamed $</$ forename $><$ genName $>\mathrm{VI}</$ genName $></$ persName>

81 36. $<$ pers.ind $><$ title $>$ Son Altesse Royale le $<$ func.ind $>$ prince $</$ func.ind $></$ title $><$ name.fi rst $>$ Rainier $</$ name.first $></$ pers.ind $>$

$<$ persName $>$ addName type="honorific" $>$ Son Altesse Royale $</$ addName $>$ le $<$ roleName type="nobility" $>$ prince $</$ roleName $><$ forename $>$ Rainier $</$ forename $></$ persName $>$

37. Le $<$ pers.ind $><$ qualifier $>$ socialiste $<$ /qualifier $><$ name.first $>$ Bertrand $</$ name.first $>$ $<$ name.last $>$ Delanoë $</$ name.last $></$ pers.ind $>$

le $<$ persName $><$ addName type="epithet" $>$ socialiste $</$ addName $><$ forename $>$ Bertrand $<$ / forename $><$ surname $>$ Delanoë $<$ /surname $><$ /persName $>$

38. le $<$ pers.ind $><$ extractor $>$ cinquième $<$ /extractor $><$ name $>$ Beatles $</$ name $></$ pers.ind $>$

le $<$ person $><$ affiliation $><$ num type $=$ "ordinal" value $=" 5$ " $>$ cinquième $</$ num $><$ org $>$ Beatles $<1$ org $></$ affiliation $></$ person $>$

Lorsqu'il n'y a pas de nom, ou lors d'une énumération, les éléments pers.ind et pers.coll peuvent être remplacés par les éléments person, listPerson et personGrp .

39. le $<$ pers.ind $><$ demonym $>$ français $</$ demonym $></$ pers.ind $>$ s'est classé quatrième

$\rightarrow$

le $<$ person $><$ nationality $>$ français $</$ nationality $></$ person $>$ s'est classé quatrième

40. $<$ pers.ind $><$ name $>$ Asterix $</$ name $></$ pers.ind $>$ et $<$ pers.ind $><$ name $>$ obelix $</$ name $></$ pers.ind $>$ ont apporté un sanglier

$<$ listPerson $><$ person $><$ persName $>$ Asterix $</$ persName $><$ /person $>$ $<$ person $><$ persName $>$ Obelix $</$ persName $></$ person $></$ listPerson $>$ ont apporté un sanglier 41. $<$ pers.coll $><$ title $>$ Monsieur $</$ title $>$ et $<$ title $>$ Madame $</$ title $><$ name.last $>$ Chirac $</$ name.last $></$ pers.coll $>$

$<$ listPerson $><$ person $><$ roleName type="honorific" $>$ Monsieur $</$ roleName $></$ person $>$ et $<$ person $><$ roleName type $=$ "honorific" $>$ Madame $<$ /roleName $>\quad<$ persName $>$ Chirac $</$ persName $></$ person $></$ listPerson $>$

42. les $<$ pers.coll $><$ demonym $>$ Français $</$ demonym $></$ pers.coll $>$ 
les $<$ personGrp $><$ nationality>Français</nationality $>$ /personGrp>

\subsection{Fonctions}

Comme dans l'exemple, les fonctions sont balisées par l'élément roleName; lorsque la fonction apparaît seule, sans nom propre, cet élément est intégré dans un élément person ou personGrp .

43. l'<func.ind $><$ kind $>$ ambassadeur $</$ kind $>$ de $<$ loc.adm.nat $>$ Turquie</loc.adm.nat $>$ en $<$ loc.adm.nat $>$ France $</$ loc.adm.nat $></$ func.ind $>$

l'<person $><$ roleName type="office" $>$ ambassadeur $</$ roleName $>$ de $<$ country $>$ Turquie $<$ / country $>$ en $<$ country $>$ France $<$ /country $><$ /person $>$

44. les $<$ func.coll $><$ kind $>$ maires $</$ kind $>$ de $<$ loc.adm.nat $>$ France $</$ loc.adm.nat $></$ func.coll $>$

les $<$ personGrp $><$ roleName type="office" $>$ maires $</$ roleName $>$ de $<$ country $>$ France $<$ / country $><$ /personGrp $>$

\subsection{Organisations}

L'élément orgName peut contenir d'autres éléments, comme un name de type role, un surname, un autre orgName, etc. On retrouve donc une équivalence comme précédemment.

45. la <org.ent $><$ kind $>$ police $</$ kind $><$ demonym $>$ française $</$ demonym $></$ org.ent $>$

$<$ org $><$ name type $=$ "role" $>$ police $</$ name $><$ nationality $>$ française $</$ nationality $></$ org $>$

46. la <org.ent $><$ kind $>$ société $</$ kind $><$ name $>$ Peugeot</name $></$ org.ent $>$

la $<$ orgName $>$ name type $=$ "role" $>$ société $</$ name $>\quad<$ surname $>$ Peugeot $</$ surname $><$ / orgName>

\subsection{Localisations}

Pour les lieux, il en est de même avec différents types ou .

47. <loc.adm.town>La Bolline</loc.adm.town> 
$<$ placeName type $=$ "city" $>$ La Bolline $</$ placeName $>$

48. <loc.adm.town>Maison Blanche</loc.adm.town $>^{11}$

$<$ placeName type $=$ "district" $>$ Maison Blanche $</$ placeName $>$

49. Le $<$ loc.adm.town $><$ name $>13 \mathrm{e}</$ name $><$ kind $>$ arrondissement $</$ kind $></$ loc.adm.town $>$

Le $<$ placeName $>$ district $><$ num type="ordinal" value="13">13e</num>arrondissement $<$ / district $></$ placeName $>$

Les lieux relatifs donnent aussi lieu à l'annotation .

50. <loc.adm.reg><qualifier>au sud </qualifier> d'<name><loc.adm.nat>Israël $</$ loc.adm.nat $></$ name $></$ loc.adm.reg $>$

$\mathrm{Au}<$ placeName $>$ offset $>$ sud d'</offset $><$ country $>$ Israël</country $></$ placeName>

Tout comme les lieux géographiques .

51. la<loc.adm.sup ><kind $>$ région </kind >de l'<name>Atlas</name></loc.adm.sup >

$\rightarrow$

la $<$ placeName $><$ offset $>$ région de $<$ /offset $><$ geogName $>$ l'Atlas $</$ geogName $></$ placeName $>$

52. le <loc.phys.geo $><$ kind $>$ désert $</$ kind $>$ de $<$ name $>$ Gobi $</$ name $></$ loc.phys.geo $>$

Le <geogName><geogFeat>désert</geogFeat $>$ de Gobi</geogName>

53. la <loc.phys.hydro>Seine</loc.phys.hydro>

la $<$ geogName type $=$ "river" $>$ Seine $</$ geogName $>$

Pour les voies de circulation, il n'est pas prévu de descripteur spécifique. Le plus simple serait d'utiliser un attribut oronym dans un élément placeName. C'est d'ailleurs ce qui est proposé par la TEI pour les bâtiments avec l'attribut building, éventuellement complété par un élément addName avec l'attribut epithet.

54. l'<loc.oro $><$ kind $>$ autoroute $</$ kind $><$ name $>$ A6 $</$ name $></$ loc.oro $>$

l'<placeName type="oronym">autoroute A6</placeName> 
l'<placeName type="building"><addName type="epithet">ancienne</addName> gare de $<$ settlement $>$ Rungis $</$ settlement $></$ placeName $>$

Pour les adresses physiques, la correspondance existe, même dans des adresses complexes, avec les éléments address et addrLine . C'est ce dernier élément que l'on utilise seul pour les numéros de téléphone . Les adresses électroniques correspondent à l'élément email et les identifiants pourraient être balisés par l'élément ident . En revanche, rien ne concerne les fréquences radio qu'on pourrait considérer à l'instar des numéros de téléphone, avec l'élément addrLine .

56. J'habite <loc.add.phys ><address-number $>15</$ address-number $><$ loc.oro $><$ kind $>$ rue $<$ / kind $>$ de $<$ name $>$ Vaugirard $</$ name $></$ loc.oro $><$ other-address-component>escalier $2</$ other-address-component $></$ loc.add.phys $>$

<address ><addrLine>15 rue de Vaugirard</addrLine> <addrLine>escalier-2</addrLine $></$ address>

110 57. mon numéro est le <loc.add.elec><name>01 698580 02</name></loc.add.elec>

mon numéro est le <addrLine>01 698580 02</addrLine>

58. mon identifiant skype est <loc.add.elec $><$ name>jean.dupont $</$ name $></$ loc.add.elec $>$

mon identifiant skype est $<$ ident type $=$ "skype">jean.dupont $<$ /ident $>$

59. $<$ prod.media $>$ Radio Bleue $</$ prod.media $>$ sur $<$ loc.add.elec $><$ name $>98.8 \mathrm{MHz}</$ name $><$ / loc.add.elec >

<orgName>Radio Bleue</orgName> sur <addrLine>98.8 MHz</addrLine>

\subsection{Productions humaines}

Comme nous le voyons dans l'exemple, il n'existe pas dans la TEI de description des productions humaines en général. La solution serait d'utiliser l'élément name complété par un attribut type et éventuellement un élément role. Pour les objets, le type pourrait prendre la valeur object ${ }^{12}$.

114 60. j'ai piloté la $<$ prod.object $><$ kind $>$ navette $</$ kind $><$ name $>$ Endeavour $</$ name $<</$ prod.object> 
j'ai piloté la <name type $=$ "object" $><$ role $>$ navette $</$ role $>$ Endeavour $</$ name $>$

61. le < prod.other $>$ <name>Monopoly</name $<$ /prod.other $>$

le <name type="object">Monopoly</name>

De même, pour les autres produits cet attribut type pourrait prendre comme valeur finance, soft , award, service, doctrine ou rule .

62. la <func.ind >gérante</func.ind $>d u \quad<$ prod.fin $><$ kind $>$ fonds $</$ kind $><$ name $>$ DWS signature court terme $</$ name $></$ prod.fin $>$ chez $<$ org.ent $>$ DWS Investments $</$ org.ent $>$ en $<$ loc.adm.nat>France</loc.adm.nat>

la $<$ person $><$ roleName type ="occupation" $>$ gérante $</$ roleName $></$ person $>$ du $<$ name type $=$ "finance" $><$ role $>$ fonds $</$ role $>$ DWS signature court terme $</$ name $>$ chez $<$ orgName>DWS Investments</orgName> en $<$ placeName type $=$ "country" $>$ France $</$ placeName>

63. le < prod.soft $><$ kind $>$ système d'exploitation $</$ kind $><$ name $>$ Unix $</$ name $></$ prod.soft $>$

le $<$ name type $=$ "soft" $><$ role $>$ système d'exploitation $</$ role $>$ Unix $</$ name $>$

64. le <prod.award $><$ name $>$ Prix Nobel</name> de <award-cat $>$ chimie $</$ award-cat $></$ prod.award>

le $<$ name type="award" $>$ Prix Nobel $<$ addName $>$ de chimie $</$ addName $></$ name $>$

65. le < prod.serv $><$ kind $>$ RER $</$ kind $><$ name $>$ B $</$ name $></$ prod.serv $>$

le $<$ name type $=$ "service" $><$ role $>$ RER $</$ role $>\mathrm{B}</$ name $>$

66. le < prod.doctr $><$ name $>$ socialisme</name $></$ prod.doctr $>$

le $<$ name type $=$ "doctrine" $>$ socialisme $</$ name $>$

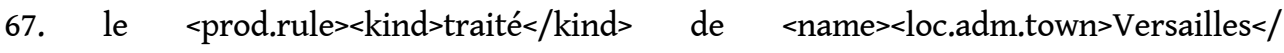
loc.adm.town $></$ name $></$ prod.rule $>$ 
le $<$ name type $=$ "rule" $><$ role $>$ traité $</$ role $>$ de $<$ settelment type $=$ "city" $>$ Versailles $<$ / settelment $></$ name>

Par contre, dans la catégorie artistique de Quaero, on pourrait plutôt utiliser l'attribut media qui permettrait de préciser le type de media utilisé, ainsi que les éléments title et author. L'élément title peut être précisé par un attribut level qui prend les valeurs $m$ (monographie), $j$ (journal), $s$ (série) ou $u$ (non publié) .

68. <prod.art $><$ name $>$ Le Baiser $</$ name $></$ prod.art $>$ de $<$ pers.ind $><$ name.last $>$ Klimt $</$ name.last $></$ pers.ind $>$

$\rightarrow$

<name media="art"><title>Le Baiser</title> de <author >Klimt</author $>$ /name>

69. <pers.ind $><$ name.first $>$ Mickaël</name.first $><$ name.last $>$ Vendetta $</$ name.last $></$ pers.ind $>$ veut quitter <prod.media><name>La Ferme des Célébrités</name $><$ / prod.media>

$<$ persName $>$ forename>Mickaël</forename>

$<$ surname $>$ Vendetta $<$ /surname $>$ / persName> veut quitter <name media="TV"><title level="s">La Ferme des Célébrités</ title $>$ /name>

70. <prod.media $><$ name $>$ Le Monde $</$ name $></$ prod.media $>$ a publié un article sur $<$ prod.art $><$ name $>1984</$ name $></$ prod.art $>$ de $<$ pers.ind $><$ name.first $>$ George $</$ name.first $>$ $<$ name.last $>$ Orwell</name.last $></$ pers.ind $>$

<orgName>Le Monde</orgName> a publié un article sur <name media="book"><title level="m">1984</title> de <author>George Orwell</author></name>

En revanche, pour l'exemple, qui n'est pas une entité nommée, il est impossible d'utiliser l'élément name. En supposant qu'il s'agisse d'une anaphore, on pourrait éventuellement utiliser l'élément $r$ avec un attribut type de valeur rule.

71. le <prod.rule $>$ qualifier>dernier</qualifier $><$ kind $>$ plan de paix $</$ kind $>$ $<$ qualifier>international </qualifier $><$ /prod.rule $>$

le $<$ rs type $=$ "rule" $><$ epithet $>$ dernier $</$ epiteth $>$ plan de paix $<$ epiteth $>$ international $<$ / epiteth $></$ rs $>$

\subsection{Quantités}

Les quantités sont bien couvertes par la TEI, avec l'élément measure. Cet élément est enrichi par les attributs quantity et unit. Lorsqu'on compte des objets, la TEI propose d'utiliser commodity... Cependant, contrairement à la proposition de Quaero, nous 
proposons de distinguer les marchandises des personnes, en utilisant les éléments person et personGrp, comme dans l'exemple .

72. $<$ amount $><$ val $>$ trois $</$ val $><$ object $>$ pompiers $</$ object $></$ amount $>$

$<$ measure quantity ="3" unit ="count" >trois $<$ person $>$ pompiers $</$ person $></$ measure $>$

Pour les quantités approximatives, la TEI propose d'utiliser l'élément extent et de placer l'élément measure à l'intérieur de celui-ci .

73. $<$ amount $><$ val $>$ quelques</val $><$ unit $>$ mètres</unit $></$ amount $>$

$<$ extent $><$ measure unit $=" \mathrm{~m} ">$ quelques mètres $</$ measure $></$ extent $>$

L'exemple utilise l'élément commodity dont nous avons parlé ci-dessus.

74./ <amount $><$ val $>$ une douzaine $</$ val $>$ de $<$ object $>$ tomates $</$ object $></$ amount $>$

$<$ measure quantity $=" 12$ unit $=$ "count" commodity $="$ tomates" $>$ une douzaine de tomates $<$ /measure $>$

Pour l'exemple, on pourraît définir une unité de valeur container applicable aussi aux cuillérées et aux cars .

$<$ measure quantity $=" 1 "$ unit $=$ "container" commodity ="tomates">une boîte de tomates $></$ measure $>$

76. $<$ amount $><$ val $>$ trois $</$ val $><$ unit $>$ cuillerées $</$ unit $>$ de $<$ object $>$ farine de châtaignes $</$ object $></$ amount $>$

$<$ measure quantity =" 3 " unit $=$ "container" commodity ="farine de châtaignes">trois cuillerées de farine de châtaignes $<$ /measure>

77. $<$ amount $><$ val $>$ un $</$ val $><$ unit $>$ car $</$ unit $>$ de $<$ object $>$ CRS $</$ object $></$ amount $>$

$<$ measure quantity =" 1 " unit ="container" $>$ un car de $<$ person $>$ CRS $</$ person $></$ measure $>$

L'élément extent permet non seulement de marquer les valeurs approximatives, comme signalé ci-dessus, mais aussi des intervalles .

14078 . $<$ amount $><$ range-mark $>$ entre $<$ /range-mark $><$ val $>$ deux $</$ val $><$ range-mark $>$ et $</$ rangemark $><$ val $>$ trois $</$ val $><$ unit $>$ kilomètres $</$ unit $></$ amount $>$ 
$<$ extent>entre <measure quantify $=" 2 "$ unit $=" \mathrm{~km} ">$ deux $</$ measure $>$ et $<$ measure quantity $=" 3$ " unit $=" \mathrm{~km} ">$ trois kilomètres $</$ measure $></$ extent $>$

L'exemple va être traité différemment. Les deux éléments amount du guide Quaero devront être remplacés par l'élément unit pour la première partie et l'élément measure pour la deuxième.

79. la distance en <amount $><$ unit $>$ kilomètres</unit $></$ amount $>$ c'est $<$ amount $><$ val $>$ trois $<$ /val $></$ amount $>$

la distance en <unit>kilomètres</unit> c'est <measure quantity ="3" unit ="km">trois</ measures

Pour l'âge d'une personne, la TEI propose un élément spécifique, age .

80. j'ai <amount $><$ val $>10</$ val $><$ unit $>$ ans $</$ unit $></$ amount $>$

j'ai <age >10 ans</age>

Il est aussi possible d'utiliser la valeur fraction de l'attribut unit .

81. <amount $><$ val $>60</$ val $><$ unit $>$ fédérations départementales $</$ unit $></ a m o u n t>$ sur $<$ amount $><$ val $>95</$ val $></$ amount $>$

$<$ measure quantity $=" 60 / 95 " \quad$ unit $=$ "fraction">60 $>$ personGrp>fédérations départementales $</$ personGrp> sur $95</$ measure>

147 82. $<$ amount $><$ extractor $>$ une $</$ extractor $>$ des $<$ val $>$ trois $</$ val $><$ object $>$ filles $</$ object $><$ / amount>

$<$ measure quantity $=" 1 / 3 "$ unit ="fraction">une des trois $<$ person $>$ filles $</$ person $><$ | measure>

\subsection{Point dans le temps}

Pour la plupart des exemples, la correspondance entre le guide Quaero et la TEI se fait bien, mais avec deux différences notables. Même si il existe des éléments month et year dans la TEI (mais non dans TimeML), il est recommandé d'utiliser l'attribut when de normalisation ${ }^{13}$ : use the when attribute to provide a normalized equivalent .

83. <time.date.abs $><$ week $>$ lundi</week> <day $>25</$ day $><$ month $>$ janvier $</$ month $>$ $<$ year $>2010</$ year $></$ time.date.abs> 
$<$ date when="2010-01-25">lundi 25 janvier 2010</date>

C'est l'attribut when qui permet la distinction entre dates relatives et dates absolues, soit par omission, soit par incomplétude de la forme normalisée . Comme ces deux notions varient selon la campagne d'évaluation et nous semblent étrangement définies, nous proposons de ne pas introduire un attribut type pour les distinguer, ce qui serait une possibilité.

84. rendez-vous <time.date.rel $>$ week>mardi</week $></$ time.date.rel>

rendez-vous $<$ date $>$ mardi $</$ date $>$

85. $<$ time.date.abs $><$ time-modifier $>$ le $</$ time-modifier $><$ day $>25</$ day $><$ month $>$ janvier $</$ month $></$ time.date.abs $>$

$<$ date when="--01-25">le 25 janvier $</$ date>

86. <time.date.abs $><$ time-modifier $>$ en $</$ time-modifier $><$ year $>300</$ year $><$ time-modifi er $>$ avant $\mathrm{JC}</$ time-modifier $></$ time.date.abs $>$

$<$ date when="B0300">en 300 avant JC</date>

L'attribut when peut être remplacé par les attributs notBefore, notAfter, from et to .

87. le <time.date.abs $><$ century $>21$ e siècle $</$ century $></$ time.date.abs $>$

le <date from ="2001" to ="2100">21è siècle</date>

Les noms de période historique sont traités de même par la TEI .

88. le <time.date.abs $><$ reference-era $>$ second empire $</$ reference-era $></$ time.date.abs $>$

le $<$ date notBefore $=" 1852-12-02$ " notAfter $=" 1870-09-04 ">$ second empire $</$ date $>$

Lorsqu'un modifieur précise une date par rapport à une autre, on utilise, comme pour les lieux, l'élément offset à l'intérieur d'un élément date. Bien que les adverbes de temps n'apparaissent pas dans les exemples de la TEI, il nous semble possible de les baliser de la même manière ${ }^{14}$.

89. <time.date.rel $><$ week>lundi</week $><$ time-modifier>prochain $</$ time-modifier $></$ time.date.rel> 
$<$ date $>$ date $>$ lundi $</$ date $><$ offset $>$ prochain $</$ offset $></$ date $>$

90. $<$ time.date.rel $><$ name $>$ hier $</$ name $>\quad<$ time-modifier $>$ matin $</$ time-modifier $></$ time.date.rel>

$<$ date $>$ date $>$ hier $<$ /date $>$ offset $>$ matin $<$ offset $>$ /date $>$

Pour les heures, le système est identique en remplaçant l'élément date par l'élément time .

91. $<$ time.hour.abs $><$ val $>$ quinze $</$ val $>\quad<$ unit $>$ heures $</$ unit $>\quad<$ val $>$ trente $</$ val $></$ time.hour.abs>

$<$ time when $=" 15: 30: 00 ">$ quinze heures trente $</$ time $>$

92. <time.hour.abs $><$ val $>3</$ val $><$ unit $>$ heures $</$ unit $><$ time-modifier $>$ du matin</timemodifier $></$ time.hour.abs>

$<$ time when ="03:00:00">3 heures du matin</time>

93. <time.hour.rel><time-modifier $>$ ce $<$ /time-modifier $>$ <name $>$ matin</name $><$ / time.hour.rel>

$<$ time notBefore="00:00:00" notAfter="12:00:00" $><$ offset $>$ ce $</$ offset $><$ time $>$ matin $<$ / times</time>

Comme pour les mesures, un intervalle est balisé par un élément extent contenant deux éléments, date ou time .

94. la réunion commencera $<$ time.hour.abs $><$ range-mark $>$ entre $<$ /range-mark $><$ val $>3<1$ val $><$ range-mark $>$ et $</$ range-mark $><$ val $>5</$ val $><$ unit $>$ heures $</$ unit $></$ time.hour.abs $>$

la réunion commencera <extent>entre <time when $=03: 00: 00 ">3</$ time $>$ et <time when $=05: 00: 00 ">5$ heures $</$ time $></$ extent $>$

\section{8. Événements}

la TEI, comme dans le guide Quaero, les évènements sont balisés par l'élément event ; dans la TEI cet élément peut éventuellement être accompagné d'un attribut when.

95. Le <event>Tour de <loc.adm.nat>France</loc.adm.nat $></$ event $>$ 
Le $<$ event $>$ Tour de $<$ country $>$ France $<$ /country $><$ /event $>$

96. le <event $>44$ e congrès de la <org.ent $>$ CFDT $<$ /org.ent $></$ event $>$

le $<$ event $><$ num type $=$ "ordinal" value $=" 44 ">44 \mathrm{e}</$ num $>$ congrès de la $<$ orgName $>$ CFDT $<$ / orgName></event>

L'élément event de la TEI (ou du guide Quaero) est ambigu - à une différence de casse près - avec l'élément du même nom, EVENT, utilisé par TimeML pour baliser les évènements, non au sens des entités nommées, mais au sens syntactico-sémantique. Cette différence de casse permet néanmoins son utilisation.

\section{Conclusion}

Dans cet article nous avons présenté les différents balisages des entités nommées utilisés dans les campagnes d'évaluation Ester et Etape, ainsi que les normes de la TEI et de TimeML. En comparant l'ensemble des exemples proposés par le guide Quaero avec la TEI, nous avons constaté que le balisage peut être effectué de manière quasiment similaire en utilisant seulement la norme TEI. Afin de stabiliser les évaluations sur le français et de permettre aussi d'utiliser les mêmes balises sur des textes d'autres langues, nous préconisons d'utiliser désormais la TEI pour l'annotation des entités nommées.

Notre système de reconnaissance et de balisage des entités nommées, CasEN, disponible en ligne ${ }^{15}$, va être modifié dans ce sens.

\section{Remerciements}

Les auteurs remercient Enza Morale de l'Inist pour sa relecture attentive de la section , ainsi que les relecteurs de la revue Corela pour leurs remarques et suggestions.

\section{BIBLIOGRAPHIE}

Artstein R., Poesio M. (2008). Inter-Coder Agreement for Computational Linguistics

Computational Linguistics. MIT Press, 34:555-596.

Chinchor N. (1997). Muc-7 Named Entity Task Definition ${ }^{16}$.

Ehrmann M. (2008). Les entités nommées, de la linguistique au TAL : statut théorique et méthodes de désambiguïsation. Thèse de doctorat, Université Paris 7 - Centre de recherche Xerox, Grenoble (XRCE).

Ester-2 (2007). Entités Nommées, Dates, heures et montants. Convention d'annotation, version $0.1 .^{17}$. 
Ferro L., Mani I., Sundheim B., Wilson G. (2001). TIDES Temporal Annotation Guidelines. Version 1.0.2 MITRE Technical Report, MTR01W0000041.

Fèvre-Pernet C., Roché M. (2005). Quel traitement lexicographique de l'onomastique commerciale? Pour une distinction Nom de marque/Nom de produit. Revue Corela.

Fort K., Nazarenko A., Rosset S. (2012). Modeling the Complexity of Manual Annotation Tasks: a Grid of Analysis. Proceedings of the International Conference on Computational Linguistics (COLING 2012).

Gross G. (2012), Manuel d'analyse linguistique. Villeneuve-d'Ascq, Presses universitaires du. Septentrion.

Lefeuvre A., Antoine J.-Y., Savary A., Schang E., Abouda L., Maurel D., Eshkol I. (2014). Annotation de la temporalité en corpus : contribution à l'amélioration de la norme TimeML. TALN 2014, Marseille.

McNamee P., Dang H. T., Simpson H., Schone P., Strassel S. M. (2010). An evaluation of technologies for knowledge base population, LREC 2010, 369-372.

Merchant R., Okurowski M., Chinchor N. (1996). The multilingual entity task (MET) overview. In Proceedings of a workshop on held at Vienna, Virginia, pages 445-447, Morristown, NJ, USA.

Nadeau D., Sekine S. (2007). A survey of named entity recognition and classification. Linguistica Investigationes, 30(1).

Pustejovsky J., Castaño J., Ingria R., Saurí R., Gaizauskas R., Setzer A., Katz G., Radev G. (2003). TimeML: A Specification Language for Temporal and Event Expressions. In Proceedings of the International Workshop of Computational Semantics.

Rosset S., Grouin C., Zweigenbaum P. (2011).Entités nommées structurées : guide d'annotation Quaero. Notes et documents Limsi :2011-04.

Santos D., Seco N., Cardoso N., Vilela R. (2006). HAREM: An Advanced NER Evaluation Contest for Portuguese. LREC 2006:1986-1991.

Sekine S. (2004). Definition, dictionaries and tagger of extended named entity hierarchy. LREC 2004, Lisbon, Portugal.

Sekine S., Eriguchi Y. (2000). Japanese named entity extraction evaluation: analysis of results. In Proceedings of the 18th conference on Computational Linguistics, Morristown, NJ, USA.

Setzer A. (2001). Temporal Information in Newswire Articles: an Annotation Scheme and Corpus Study, PhD dissertation, University of Sheffield.

Text Encoding Initiative Consortium (2012). TEI Lite: Encoding for Interchange: an introduction to the TEI Final revised edition for TEI P $5{ }^{18}$.

Text Encoding Initiative Consortium (2015). TEI P5: Guidelines for Electronic Text Encoding and Interchange ${ }^{19}$.

Tran M. (2006). Prolexbase. Un dictionnaire relationnel multilingue de noms propres : conception, implantation et gestion en ligne. Thèse de doctorat, Université François-Rabelais de Tours.

Tran M., Maurel D. (2006), Prolexbase : Un dictionnaire relationnel multilingue de noms propres, Traitement automatique des langues, vol. 47(3):115-139. 


\section{NOTES}

1. http://www.cnts.ua.ac.be/conll2002/ner/

2. http://www.cnts.ua.ac.be/conll2003/ner/

3. Même trois si l'on compte la campagne Ester-1 pour laquelle la tâche d'extraction d'entités nommées est restée à diffusion restreinte.

4. http://www.tei-c.org/

5. Voir cependant (Lefeuvre, 2014) pour une discussion sur la norme TimeML.

6. Tous les exemples cités dans cet article sont extraits des guides d'annotations concernés.

7. Les auteurs de l'article ont bien conscience de la difficulté que représente la constitution d'un tel guide. Ces remarques doivent être prises comme des critiques constructives pour des annotations futures.

8. http://www.quaero.org/

9. L'exemple du guide pour rendez-vous mardi prochain comporte les balises time.date.rel, contrairement au contexte, ce qui nous conforte dans l'idée que cette règle est difficile à soutenir.

10. Lorsqu'un exemple semblait redondant étant donné notre propos, nous l'avons omis pour ne pas rallonger inconsidérément cet article.

11. Une note du guide Quaero précise qu'il s'agit d'un quartier de Paris.

12. Il semble difficile de penser que le Monopoly n'est pas un objet. La catégorie prod.other est étrange ici. Comme le guide Quaero précise, sans les exiger et donc sans les mettre dans les exemples, qu'il est possible de créer des sous-types véhicule, aliment, vêtement, médicament et arme, cette catégorie prod.other devrait plutôt correspondre à une catégorie prod.object.other, ce qui justifie notre choix.

13. Cette normalisation suit l'ISO 8601.

14. Ce qui serait plus logique que de baliser un adverbe par l'élément name comme le demande le guide Quaero.

15. http://tln.li.univ-tours.fr/Tln_CasEN.html

16. http://www.itl.nist.gov/iaui/894.02/related_projects/muc/proceedings/ne_task.html

17. http://www.afcp-parole.org/camp_eval_systemes_transcription/docs/

Conventions_EN_ESTER2_v01.pdf

18. http://www.tei-c.org/release/doc/tei-p5-exemplars/pdf/tei_lite.doc.pdf

19. http://www.tei-c.org/release/doc/tei-p5-doc/en/Guidelines.pdf

\section{RÉSUMÉS}

Dans cet article, nous comparons les campagnes existantes d'annotation d'entités nommées avec la TEI. Après une analyse détaillée, nous préconisons une utilisation de la norme TEI afin d'uniformiser et d'adapter les méthodes d'annotation des entités nommées utilisées, notamment celles des campagnes Etape et Ester, menées dans le contexte francophone. 
This paper deals with French Named Entity shared tasks. We compare the elements defined in the different guidelines with those defined by the TEI Consortium. We suggest to use the TEI guideline, which is shared by a large community in the world, in future Named Entity tasks.

INDEX

Keywords : Named Entity, tags, evaluation campaign, Etape, Ester, TEI

Mots-clés : Entités nommées, annotations, campagnes d'évaluation, Etape, Ester, TEI

\section{AUTEURS}

\section{SOLENN LE PEVEDIC}

Université François Rabelais de Tours

Laboratoire d'Informatique

DENIS MAUREL

Université François Rabelais de Tours

Laboratoire d'Informatique 